\title{
Eine Gleichung mit zwei Unbekannten: Johannes Widekindis Exzerpte aus Petrus Petrejus
}

\author{
Arsenij A. Vetushko-Kalevich \\ Lunds Universitet, Språk- och litteraturcentrum, \\ Helgonabacken 12, Box 201, 22100 Lund, Sverige; arsenii.vetushko-kalevich@klass.lu.se \\ St. Petersburg State University, \\ 7-9, Universitetskaya nab., St. Petersburg, 199034, Russian Federation; st020343@student.spbu.ru
}

For citation: Arsenij A. Vetushko-Kalevich. Eine Gleichung mit zwei Unbekannten: Johannes Widekindis Exzerpte aus Petrus Petrejus. Philologia Classica 2018, 13(1), 116-130. https://doi.org/ $10.21638 / 11701 /$ spbu20.2018.109

The scale and character of the Swedish historian Petrus Petrejus' influence on Historia Belli Sveco-Moscovitici Decennalis by his countryman Johannes Widekindi have not been subject to systematical research; only a few of the loaned passages have been identified as such by Helge Almquist and the editors of the Russian translation of Widekindi. The general evaluation of Petrejus' importance for Widekindi as formulated by the latter ones is nevertheless somewhat exaggerated. This article contains a list of the loans and a discussion of the problems that emerge when distinguishing the loans from Petrejus from the loans from the other sources (Stanisław Kobierzycki, Axel Oxenstierna, geographical and documentary sources). The analysis points out that Widekindi has not used the manuscript of Conrad Bussow's chronicle. Finally, the mechanism of Widekindi's work with Petrejus is specified. Despite being acquainted with the German version, he usually resorted to the Swedish one. Some details of Widekindi's rendering of the information given by Petrejus demonstrate that when paraphrasing (and not quoting word for word, as on three occasions) Petrejus, the Latin text of Widekindi is the source text for his Swedish. So two steps of the work may be discerned: first Widekindi paraphrased Petrejus in his Latin draft, and then he added to the Swedish translation some fragments of the Swedish text by Petrejus.

Keywords: Time of Troubles, Ingrian War, Swedish Neo-Latin, 17th century historiography, Rossica, Johannes Widekindi, Petrus Petrejus.

In der Reihe der Ereignisse, die in der russischen Zeit der Wirren stattfanden, spielt die Auseinandersetzung zwischen Russland und Schweden, als der Ingermanländische Krieg bekannt, eine ziemlich bescheidene Rolle, sowohl in den Quellen des 17. Jahrhunderts als auch in dem geschichtlichen Gedächtnis von heute. Selbst in Schweden erschien davon nur ein ausführlicher Bericht (und erst ein halbes Jahrhundert später), nämlich das Werk von dem Reichshistoriografen Johannes Widekindi. Es liegt allen modernen Forschungen zugrunde, die mit dem Ingermanländischen Krieg zu tun haben.

Widekindis Buch erschien 1671 auf Schwedisch als Thet Swenska i Ryssland Tijo åhrs Krijgz-Historie und 1672 auf Latein als Historia Belli Sveco-Moscovitici Decennalis. Die Lateinische Ausgabe ist jedoch keineswegs eine Übersetzung der Schwedischen: in einem Brief zu seinem Patron, Reichskanzler Graf Magnus Gabriel De la Gardie, berichtet Widekindi, dass er die Geschichte zuerst auf Latein verfasste und danach in Eile ins

(C) St. Petersburg State University, 2018 
Schwedische übersetzte. ${ }^{1}$ Die Beschaffenheit der zwei gedruckten Versionen, besonders im Vergleich mit Widekindis bekannten lateinischen Quellen, zeugt davon, dass die schwedische wirklich zum grossen Teil aus Übersetzungen besteht, während die lateinische eine leichte Bearbeitung des (verlorengegangenen) Entwurfs ist. ${ }^{2}$ Es gibt jedoch Fragmente, die in der schwedischen Version von den schwedischen Urkunden wörtlich abgeschrieben sind. ${ }^{3}$ Die Frage vom Status der zwei Texte kann also nur im Vergleich mit dem Text der Quellen in jedem einzelnen Fall beantwortet werden.

Was sind dann die Quellen, die Widekindi benutzt? Betreffend der schwedischen Kriegshandlungen im Nord-Westen des heutigen Russlands schöpft er sein Wissen aus schwedischen Urkunden, die teilweise bis heute im Reichsarchiv in Stockholm aufbewahrt werden. Was andererseits die russisch-polnischen Verhältnisse betrifft, stützt er sich meistens auf lateinische Schriften, nämlich einen Bericht vom schwedischen Reichskanzler Axel Oxenstierna und die Historia Vladislai des polnischen Geschichtsschreibers Stanisław Kobierzycki. Die Details der älteren Geschichte und der Geographie Russlands sind oft ebenfalls den lateinischen Schriften entlehnt.

Gab es in Schweden zu Widekindis Zeit grosse, gedruckte Rossica-Werke von schwedischen Verfassern? Eigentlich nur zwei. Das eine, Historia de gentibus septentrionalibus (1555) von Olaus Magnus, wo besonders das elfte der 22 Bücher reich an Russland-bezogenen Informationen ist, scheint Widekindi zu ignorieren; es ist nicht auszuschliessen, dass er sie für veraltet hielt (fast alle bekannten Quellen, die Widekindi unmittelbar benutzte, waren zur Zeit seiner eigenen Publikation nicht mehr als 100 Jahre alt). ${ }^{4}$ Ein anderes Werk, das zu Verfüging stand, war hingegen für Widekindis Stoffgebiet höchst relevant, nämlich die Chronik von Petrus Petrejus, der sich gerade am Beginn des 17. Jahrhunderts in Russland aufhielt.

Einige Worte seien über diesen und sein Werk im Allgemeinen gesagt. Petrus Petrejus wurde ca 1570 in Uppsala geboren; sein Vater war der zukünftige Bischof Petrus Benedicti. Seine Universitätsstudien verbrachte Petrejus in Marburg. Er soll der erste Schwede sein, der über einen mathematischen Gegenstand dissertierte. Wie er nach Russland geriet, ist nicht klar. Er befand sich in Moskau 1601-1605 und scheint dem tsarischen Hofe nahe gestanden zu haben. Nach seiner Rückkehr nach Schweden liess er 1608 einen kurzen Bericht von den turbulenten Ereignissen der letzten Jahre erscheinen, Een wiss och sanfärdigh berättelse, om några förandringar, som $j$ thesse framledne åhr, vthi storfurstendömet Muskow skedde äre. ${ }^{5}$ Seine Erfahrung wurde von Karl IX. hoch geschätzt,

1 Vetushko-Kalevich 2016.

2 Beispiele davon s. in Vetushko-Kalevich 2018.

3 Z. B. Evert Horns Bericht, datiert 23 Mai 1610 (Svenska Riksarkivet, Handlingar rörande ryska kriget), ist in Widekindi 1671, 177-181, 183 wiedergegeben, und das Protokoll der Verhandlungen in Viborg am 28. August 1613 (Svenska Riksarkivet, Diplomatica Muscovitica, vol. 17, fol. 31-35) ist teilweise in Widekindi 1671, 505-514 abgeschrieben.

${ }^{4}$ Er erwähnt zwar Wandalia von Albert Krantz (gedruckt 1517), aber der Hinweis kommt aus zweiter Hand (s. Vetushko-Kalevich 2017, 181). Die einzige Ausnahme ist also Europa von Aeneas Sylvius Piccolomini (verfasst in der Mitte des 15. Jahrhunderts), und auch diese vermutlich in der Ausgabe von 1571 (ebd. 180 n. 9).

${ }^{5}$ Ob Widekindi auch diese erste Schrift von Petrejus kannte, ist nicht sicher. Es gibt davon nur eine mögliche Spur, nämlich die gemeinsame Behauptung, dass der erste falsche Dmitri seine heimtückischen Pläne, Russland zum Katholizismus zu bekehren, seinem Marschall Wassili Mosalski entschleierte (Petrejus 1997, 134; Widekindi 1671, 30-31; Widekindi 1672, 27; s. Videkind 2000 (Видекинд, Ю.История десятилетней шведско-московитской войны. Москва, Памятники исторической мысли, 2000), 
und Petrejus wurde in den Jahren 1607-1610 noch mehrmals nach Moskau geschickt. Im Frühling 1611 treffen wir ihn in Ivangorod, wo er im königlichen Auftrag mit dem dritten falschen Dmitri zu verhandeln - und zu sehen, ob dieser dem ersten falschen Dmitri ähnlich ist - versucht. ${ }^{6}$ Endlich kehrt Petrejus nach Schweden zurück und verfasst ein grosses Werk über Russland, das 1615 unter dem Titel Regni Muschovitici Sciographia ${ }^{7}$ auf Schwedisch (mit lateinischen Marginalien) erschien. Das Werk besteht aus sechs Teilen: im ersten wird die Geographie von Russland erörtert, im zweiten seine Geschichte, und die weiteren vier kürzeren Teile handeln von russischen Zeremonien, Kriegswesen, Alltagsleben und Religion.

Sowohl die diplomatische Wirksamkeit von Petrejus als auch sein umfangreiches Werk wurden von der schwedischen Regierung hoch geschätzt, und er wurde mit einem Landgut belohnt, das er im Folgenden in seinem Namen zu erwähnen pflegte: "Petrus Petrejus de Erlesunda”. Einige Jahre später wurde er jedoch genötigt, Schweden zu verlassen und sich nach Deutschland zu begeben. Dort liess er seine Sciographia ins Deutsche übersetzen, und sie erschien 1620 unter dem Titel Historien vnd Bericht von dem Grossfürstenthumb Muschkow. Die deutsche Version ist hie und da etwas gekürzt und terminologisch etwas weniger präzis, ${ }^{8}$ aber sie ist auch aktualisiert: der zweite Teil ist dadurch vermehrt, dass er jetzt auch den Frieden von Stolbowo und seine Ratifikation enthält. Petrejus starb 1622 an der Pest, kurz nach seiner Heimkehr nach Schweden. ${ }^{9}$

Widekindi erwähnt Petrejus mehrmals, ${ }^{10}$ doch nur als eine handelnde Person - er will ja den Leser mit Quellenhinweisen nicht überlasten. ${ }^{11}$ Bei seiner ersten Erwähnung ist Petrejus aber immerhin auch mit der Charakteristik "Chronici Moschovitici scriptor" (in der schwedischen Ausgabe etwas vager: "Pehr Pehrsson (som hafwer Rysslandz lägenheet beskrifwit)") versehen. Doch erst am Ende der lateinischen Version, im Anhang über Nowgorods Lage und Geschichte, der unausgereift ist und ein Fragment aus dem Entwurf darzustellen scheint, stösst man auf einen Marginalhinweis: "Petrei.p. 2. fol. 74". Der Hinweis ist in gewisser Hinsicht irreführend; wir werden ihn etwas ferner berühren.

Wie gross ist eigentlich der Einfluss von Petrejus auf seinen späteren Kollegen? Wassili Geiman, einer der Urheber der russischen Übersetzung von Widekindi, behauptet (meine Übersetzung): "Was Russlands Innenleben, Geschichte, geographische und derartige Auskünfte betrifft, benutzt Widekindi ziemlich augenfällig "Historien von dem Grossfürs-

567 n. 55). Diese Nachricht kann gleichwohl der Schrift Tragoedia Demetrio-Moscovvitica von Matthias Schaum entlehnt sein (Schaumius 1614, C iij; Schaum benutzt vermutlich Petrejus), dessen Formulierung etwas näher zu Widekindis steht.

${ }^{6}$ Eine ausführliche Studie von Petrejus Reisen nach Russland 1607-1613 stellt Tolstikov 2012a (Толстиков, А. В.Пётр Петрей в России (1601-1613 гг.). Studia Slavica et Balcanica Petropolitana 2012, $12,129-142), 132-139$ dar.

7 Das Wort "sciographia", das hier und anderswo im Neulatein (nicht zuletzt in den Titeln wissenschaftlicher Werke) in der Bedeutung "Umriss" verwendet wird, verdankt seine Entstehung einer Fehllesung in Vitr. De arch. I, 2, 2 (Helander 2004, 171).

8 Betreffend Terminologie (aber sonst etwas oberflächlich) s. Tolstikov 2011 (Толстиков, А. В. Зачем переводить "Regni Muschovitici sciographia" Петра Петрея со шведского языка. Средние века 2011, 72, 175-186).

9 Fernere Einzelheiten über Petrejus Leben und Werke s. in Tarkiainen 1971-1972, Tarkiainen 19951997, Tarkiainen 1997, Tolstikov 2012a.

10 Widekindi 1671, 156 (= Widekindi 1672, 127); Widekindi 1672, 143; Widekindi 1671, 293 (= Widekindi 1672, 231); Widekindi 1671, 343 (= Widekindi 1672, 279); Widekindi 1671, 465 (= Widekindi 1672, 374).

11 Vetushko-Kalevich 2017, 178-179. 
tenthumb Muschkow" von seinem Landsmann Petrus Petrejus". ${ }^{12}$ Eine solche Einschätzung ist übertrieben: von den nach meiner Rechnung ungefähr 50 Stellen, wo Widekindi etwas über die Geographie Russlands sagt (oft sind es einfache Angaben über die Entfernung verschiedener Orte voneinander), stammen nur ein Dutzend von Petrejus, und den drei grössten Exkursen - von Pskov, von den Kosaken und von dem oben erwähnten Anhang über Nowgorod - liegen andere Quellen zugrunde. ${ }^{13}$

Es scheint angebracht, eine Liste der Stellen, die mit zureichender Sicherheit als Petrejus entlehnte oder teilweise entlehnte gelten können, anzuführen - in der Ordnung, in der sie in Widekindis Texten vorkommen:

I. 9: die Geschichte von Gustav, dem Sohn des König Eriks XIV.

II. 2: der Briefwechsel zwischen Wassili Schujskij und Sigismund

II. 3-4: der Bolotnikow-Aufstand

II. 6: die Anfänge des Zuges des zweiten falschen Dmitri (einige Details, sonst nach Kobierzycki)

II. 9: die Erfolge des zweiten falschen Dmitri im nördlichen Russland

III. 6 und VI. 12: Notizen über die Stadt Jaroslawl ${ }^{14}$

III. 9: Notizen über die Stadt Torschok

III. 11: Notizen über die Wolga

III. 20: Notizen über Aleksandrowskaja Sloboda

III. 25: Notizen über Troize-Sergijew-Kloster ${ }^{15}$

III. 26: der Abschied von dem zweiten falschen Dmitri und Marina Mniszech (nur ein Detail, nämlich Dmitris Tränen — sonst nach Kobierzycki); Notizen über die Stadt Kaluga; ${ }^{16}$ Marina Mniszechs Reise von Jan Sapieha zum zweiten falschen Dmitri

III. 28: Ergebnisse des Feldzuges von De la Gardie und der Willkommen des schwedischen Heeres in Moskau (in Kombination mit Kobierzycki) ${ }^{17}$

IV.3: der Abstand zwischen Moschaisk und Wjasma

IV. 6: Notizen über die Stadt Moschaisk

IV. 14: der Gemütszustand in Moskau nach der Schlacht von Kluschino

V.2: Notizen über die Festung Ladoga

V.5: die Ermordung des zweiten falschen Dmitris (in Kombination mit Kobierzycki) ${ }^{18}$

V. 11: Notizen über die Festung Nöteborg ${ }^{19}$

VI. 13: Notizen über die Stadt Wologda und den Fluss Suchona ${ }^{20}$

Anhang über Nowgorod (einige Details)

Die Liste ist nicht unproblematisch, da es nicht immer einfach ist, die Entlehnungen aus Petrejus von den Entlehnungen aus anderen Quellen zu unterscheiden. Ein Kommentar ist nötig, und ich möchte hier einige m. E. wichtigste Aspekte dieses Problems kurz erörtern.

12 Geiman 2000 (Гейман, В.Г.Сочинение Ю.Видекинда как источник по истории Смутного времени, in: Videkind 2000, 509-520), 518.

13 Vetushko-Kalevich 2017, 179-182.

14 Videkind 2000, 599 n. 370; Kovalenko et al. 2000 (Коваленко, Г. М., Хорошкевич, А. Л., Плигузов, А. И. Апология Юхана Видекинда, in: Videkind 2000, 521-561), 545.

15 Videkind 2000, 579 n. 172.

16 Videkind 2000, 579 n. 177.

17 Almquist 1907, 176 n. 3.

18 Almquist 1907, 220 n. 2.

19 Almquist 1907, 244 n. 1.

20 Videkind 2000, 600 n. 376. 


\section{Petrejus oder Stanisław Kobierzycki?}

Der polnische Geschichtsschreiber, der Widekindis bedeutendste literarische Quelle darstellt, hat Petrejus viel benutzt, und es erweckt manchmal den Anschein, dass Widekindi sich auf Petrejus stützt, wo man es eigentlich mit einer Entlehnung aus Kobierzycki zu tun hat. Letzten Endes ist die Antwort hier jedoch immer unzweideutig, denn die sprachliche Form der lateinischen Version von Widekindi (von der die schwedische in diesen Fragmenten bloss eine, manchmal verkürzte, Übersetzung ist) folgt immer unverkennbar dem Text von Kobierzycki, der ja auch lateinisch ist. ${ }^{21}$ Ein interessanter Fall liegt am Ende des 3. Buches vor: die Beschreibung des Empfanges von den Schweden in Moskau ist teilweise aus Petrejus entlehnt, teilweise andererseits aus Kobierzycki, dessen Worte allerdings aus einem ganz verschiedenen Zusammenhang herausgelöst sind. ${ }^{22}$

\section{Petrejus oder Axel Oxenstierna?}

Der handschriftliche Bericht des schwedischen Reichskanzlers über die schwedischpolnischen Beziehungen am Beginn des 17. Jahrhunderts ${ }^{23}$ liegt dem ganzen 1. Buch von Widekindi zugrunde. ${ }^{24}$ Widekindis lateinischer Text stellt hier, wie im Fall mit Kobierzycki, nur eine leichte Variation der Quelle dar. Ein Beispiel der Kombination von Oxenstierna und Kobierzycki sei hier angeführt:

Sic deluso Gudenovio personatus Demetrius, subnixus copiis Sandomiriensibus et plurimis voluntariis Polonis, qvi aut odio gentis Moschicae, aut spe praedae pellecti, exercitum ejus auxerant, confinia ingressus, paratam seditiosorum trahebat manum (AOSB I:1, 245).

$+$

Tum... crebrescit, superstitem esse Demetrium... apud imperitissimi cujusque promtas aures, aut rursum apud turbidos, saevaque Itodun $i^{25}$ tyrannide fessos, eoque nova cupientes. Vulgabatur interim per Moschoviam credebaturque, finitimas arces passim metu, passim sponte, adventantis novi Ducis accepisse imperium (Kobierzycki 1655, 58-59).

$=$

Et deluso Gudenovio personatus Demetrius primo subnixus copiis Sandomiriensibus et plurimis Polonis, qui aut studio novitatis, aut odio gentis, aut spe praedae pellecti exercitum ejus auxerant, confinia ingressus paratam seditiosorum manum invenit, quae passim metu, passim sua sponte adventantis novi Ducis accepit imperium (Widekindi 1672, 23-24). ${ }^{26}$

Leider gibt es jedoch in der bewahrten Abschrift des Berichtes eine Lakune, und der entsprechende Teil in Widekindis Text erstreckt sich von der Mitte des vierten bis zum

${ }^{21}$ Die Auskunft über die polnische Übergabe von Smolensk in III. 14 stammt also nicht, wie man im Kommentar zur russischen Übersetzung (Videkind 2000, 575 n. 135) annimmt, aus Petrejus, sondern aus Kobierzycki 1655, 78.

22 Kobierzycki 1655, 181-182.

${ }^{23}$ Historica Relatio rerum anno 1625 et seqventi huc usque gestarum inter Regna Sueciae et Poloniae (der bewahrte Teil ist herausgegeben: AOSB I:1, 239-246).

${ }^{24}$ Für weitere Einzelheiten s. Vetushko-Kalevich 2017, 183-184. Die Annahme, dass Widekindis 1. Buch sich zum grossen Teil auf Petrejus stützt (Kovalenko et al. 2000, 541), ist also falsch.

25 D. h. Godunow. Die Benennung "Itodunus", die auch Widekindi aus Kobierzycki manchmal entlehnt, geht offenbar zu einer Fehllesung von 'H' als 'It' zurück.

${ }_{26}$ Die Annahme, dass 'die vorbereitete Bande der Rebellen' auf die Benutzung von Petrejus hinweist (Videkind 2000, 566 n. 43), trifft also nicht zu. 
Ende des neunten Kapitels. Ein paar Einschaltungen von anderen Quellen (nämlich Kobierzycki und Chyträus) lassen sich zwar in diesem Teil identifizieren, aber den exakten Ursprung der meisten Einzelheiten darin wird man nie mit Sicherheit entdecken können.

Petrejus, den auch Oxenstierna kannte und vermutlich las, ist in dieser Hinsicht, soweit ich sehe, an zwei Stellen zu beachten. Im Kapitel 7 erwähnt Widekindi, dass man annimmt, der Zar Fjodor Iwanowitsch sei von Boris Godunow vergiftet worden. So berichtet es auch Petrejus, ${ }^{27}$ und die Herausgeber der russischen Übersetzung halten ihn auch hier für Widekindis Quelle. ${ }^{28}$ Letzten Endes kann es so sein, aber es liegt nahe, dass Oxenstierna, dessen Einstellung zu Godunow eine deutlich negative ist, diese Annahme in dem verlorenen Teil wiederholte - wie es auch Matthias Schaum macht, ${ }^{29}$ und auch diesen könnte Widekindi wohlgemerkt benutzt haben (s. oben).

Anders verhält es sich mit dem zweiten Abschnitt, über Gustav Eriksson im Kapitel 9. Widekindi berichtet zuvor von dem Besuch der polnischen und der schwedischen Gesandten in Moskau. Danach heisst es, etwas unerwartet: "Hic etiam est ille qui Gustavum Regis Erici exulem filium..." - es folgen einige Zeilen über Gustavs Aufenthalt in Russland, die wiederum von den Worten "Vix dimissi erant Legati..." abgelöst sind. Die Episode ist also eine ungelenkige Einschaltung der Auszüge aus einer Quelle in den Abschnitt, der von einer anderen Quelle abgeschrieben ist. Dass die letztere hier Oxenstierna und die erstere Petrejus ist, ist m. E. fast unzweifelhaft: der Text der Episode steht den Einzelheiten bei Petrejus auffällig nahe (obschon dieser viel ausführlicher ist), ${ }^{30}$ und die Episode mit den Gesandten passt gut zu Oxenstiernas Hauptinteressen in dem Bericht, da er in erster Linie von den schwedisch-polnischen Beziehungen erzählt und ausserdem an den diplomatischen Einzelheiten, trotz konsequenter Kürze, nicht vorbeigeht (in dem bewahrten Teil sind z. B. "lites" zwischen Karl IX. und dem ersten falschen Dmitri erwähnt).

\section{Petrejus oder Konrad Bussow?}

Petrejus selbst benutzte für seine Chronik ein Werk von dem deutschen Offizier Konrad Bussow, ${ }^{31}$ und inhaltsmässig haben auch bei diesem fast alle nicht-geographische Abschnitte, die von Widekindi aus Petrejus entlehnt sind, eine Entsprechung. Manchmal fragt man sich, ob Widekindi nicht selbst Bussow benutzt haben könnte: hatte Petrejus eine Handschrift von Bussow, so ist es nicht ganz auszuschliessen, dass auch sein späterer Kollege zu dieser Handschrift Zugang hatte. Bei einem detaillierten Vergleich aller drei Texte (oder eigentlich fünf, wenn man beide Versionen von Petrejus und beide Versionen von

27 Petrejus 1615, II, 110; Petrejus 1620, 263.

28 Videkind 2000, 564 n. 31.

29 Schaumius 1614, B iij.

30 Petrejus 1615, II, 119-122; Petrejus 1620, 272-275. Petrejus verfasste auch einen längeren Bericht über diesen merkwürdigen Abstämmling der schwedischen Königsfamilie (Svenska Riksarkivet, Manuskriptsamlingen, vol. 223, fol. 21r-26v); s. Tarkiainen 1971-1972, 258 und detaillierter Tolstikov 2012b (Толстиков, А. В. «Реляция о Густаве, сыне короля Эрика» и Пётр Петрей, in: Грани сотрудничества: Россия и Северная Европа: Сборник научных статей. Петрозаводск, Издательство ПетрГУ, 2012, 104-121). Diese Schrift wurde 1913 von Henri Biaudet als anonym herausgegeben (Biaudet 1913, 112-120); dass die von ihm benutzte Dresdener Handschrift mit einer Stockholmer, die man Petrejus ziemlich sicher attribuiert, identisch ist, ist erst vor kurzem von Alexandr Tolstikov festgestellt worden.

31 Tarkiainen 1971-1972, 263-265. Über Bussows Person und Werk s. Smirnov 1961 (Смирнов, И. И. Конрад Буссов и его хроника, in: Буссов, К. Московская хроника 1584-1613. Москва-Ленинград, Издательство Академии Наук СССР, 1961, 5-62) und Orchard 1978 mit weiterer Bibliographie. 
Widekindi einrechnet) erweist sich allerdings in allen relevanten Abschnitten, dass Widekindi näher zu Petrejus als zu Bussow steht. Bussow nennt z. B. Godunows Begleitbrief für Gustav Eriksson nicht, ${ }^{32}$ sagt kein Wort von Sigismunds Versprechen im Briefwechsel mit Wassili Schujskij, den Waffenstillstand einzuhalten, ${ }^{33}$ und die Einwohner von Putiwl sammeln in Bussows Bericht am Beginn des Bolotnikow-Aufstandes ihre Kriegsmacht von "dem weiten Felde", während Petrejus - und nach ihm Widekindi - dieses Feld als "Tatariske Ödhemarcker" / "Tartarische wüsteney" resp. "Tartariske Gebieter" / "Tartarorum desertis" bezeichnen. ${ }^{34}$ Auch die Orthographie der russischen Eigennamen steht bei Widekindi näher zu Petrejus als zu Bussow: Grigorij Schakhowskoj heisst bei Widekindi "Schac(h)opski" / "Schacoppius", bei Petrejus "Schac(h)opski", bei Bussow doch "Sachof(f) $s k i$ "; 35 die Stadt Jaroslawl ist in einem und demselben Zusammenhang bei Widekindi "Jaroslau" genannt, bei Petrejus "Jaroslaw", bei Bussow "Ierislavia". ${ }^{66}$

Ein umgekehrtes Verhältnis habe ich nirgends beobachtet. Man kan also ziemlich sicher feststellen, dass Widekindi Bussow nicht benutzte.

\section{Petrejus oder andere historisch-geographische Quellen?}

Hier stossen wir auf die vielleicht grösste Schwierigkeit: während der Kreis der Quellen für den Ingermanländischen Krieg ziemlich klein und für die ganze Zeit der Wirren mehr oder weniger überschaubar ist, können historisch-geographische Einzelheiten über Russland im 17. Jahrhundert von einer Unmenge an Schriften stammen, sowohl gedruckten als auch handschriftlichen. Die Details, die man in diesen Schriften findet, sind zu verschiedenem Grade stereotyp. Für das 16. Jahrhundert hat man ja auch einen "Stammbaum" der Rossica-Literatur gezeichnet. ${ }^{37}$ Man kann deswegen nur vorsichtig von Widekindis Entlehnungen aus Petrejus sprechen - die geographischen Notizen, die denen bei Petrejus ähnlich sind, könnten auch aus anderen Quellen stammen. Das gilt zum Beispiel für den Exkurs über den Don und den Dnepr (VII.5). In beiden Fällen gibt Petrejus nur eine ganz knappe Auskunft, Widekindis Einzelheiten sind zahlreicher und gehen mindestens zum Teil auf andere (bisher nicht festgestellte) Quellen zurück; ${ }^{38}$ was den Dnepr betrifft, kann man die Benutzung von Petrejus schlicht ausschliessen, denn alles, was er davon sagt, findet man auch in dem handschriftlichen Traktat vom deutsch-schwedischen Diplomaten Philipp Crusius von Krusenstiern, ${ }^{39}$ der Widekindi nachweislich bekannt war. ${ }^{40}$ Ausserdem nennt Krusenstiern, wie auch Widekindi, die Entfernung des Dorfes Dneprowskoje von Moskau, und die beiden begnügen sich mit zwei Städten, an denen der

${ }^{32}$ Petrejus 1615, II, 119; Petrejus 1620, 273; Widekindi 1671, 19; Widekindi 1672, 18. Vgl. Bussow 1961, 209.

${ }_{33}$ Petrejus 1615, II, 207; Petrejus 1620, 376; Widekindi 1671, 38; Widekindi 1672, 33. Vgl. Bussow 1961, 263.

34 Bussow 1961, 266; Petrejus 1615, II, 212; Petrejus 1620, 381; Widekindi 1671, 34; Widekindi 1672, 40.

35 Widekindi 1671, 40-41; Widekindi 1672, 34-35; Petrejus 1615, II, 210 etc.; Petrejus 1620, 379 etc.; Bussow 1961, 265 etc.

36 Widekindi 1672, 44 (der Abschnitt ist in der schwedischen Version abwesend); Petrejus 1615, II, 240; Petrejus 1620, 418; Bussow 1961, 286.

37 Mund 2003, 396-397.

38 Vgl. die vorsichtigen Kommentare in Videkind 2000, 602 nn. 399, 402.

39 Gründtliche Nachricht und Anweisungh Worinnen die Russische Handlung fürnemblich bestehe (Uppsala Universitetsbibliotek, L 161, fol. 10-34); die Worte über den Dnepr findet man auf fol. 14.

40 Vetushko-Kalevich 2017, 183. 
Fluss vorbeiläuft, nämlich Smolensk und Kiew, während Petrejus noch Wjasma, Dorogobusch und Orscha erwähnt. ${ }^{41}$

Eine geographische Quelle, die Petrejus und Widekindi vermutlich gemein war, ist Sigismund von Herbersteins berühmtes Werk Rerum Moscoviticarum Commentarii. Diesem scheint Widekindi die Notizen über Twer (III. 11) und Pereslawl-Salesski (III. 20) zu verdanken, die von den entsprechenden Abschnitten bei Petrejus an manchen Punkten auffällig abweichen. Der Ursprung der Parenthese über die Fürstin Olga in der Beschreibung von Pskow (VI. 9), die sowohl an die Worte von Herberstein als auch die von Petrejus erinnert, kann fraglich bleiben, obwohl einiges für Petrejus spricht. ${ }^{42}$

\section{Petrejus oder die Urkunden?}

Sooft eine solche Frage auftaucht, und das ist der Fall bei einer Reihe von Episoden, in die die Schweden 1611-1618 selbst verwickelt waren, scheint es natürlich, zu vermuten, dass Petrejus und Widekindi unabhängig voneinander Zugang zu den Urkunden hatten. Vergleicht man z. B. die Beschreibung der Zusammentreffen von Jakob De la Gardie und Wassili Buturlin am 6. und 9. Juni 1611, wird es ganz klar, dass die beiden demselben Protokoll folgen. ${ }^{43}$ Dasselbe gilt für den Bericht über die Bestätigung des Friedens von Stolbowo in Moskau und in Stockholm. ${ }^{44}$ Schwieriger verhält es sich mit den Einzelheiten im Aufkommen des dritten falschen Dmitri (V.9) und der Bestürmung von Nowgorod (V. 12): ${ }^{45}$ einige davon erinnern an den Text von Petrejus, aber Widekindi standen sicher auch andere, dokumentarische Quellen zur Verfügung.

Wenden wir uns jetzt den Stellen zu, wo Petrejus unzweifelhaft oder fast unzweifelhaft Widekindis Quelle ist, bleiben zwei ziemlich natürliche Fragen, die wir noch nicht beantwortet haben: 1) Welche Version von Petrejus' Werk benutzte Widekindi? 2) In welcher Sprache schrieb Widekindi die Abschnitte zuerst, die auf Petrejus basieren?

41 Übrigens scheint der Exkurs aus einer Reihe von verschiedenen Quellen kompiliert zu sein. Ausser den Krusenstiern-Exzerpten gibt es in Widekindis Text Ausdrücke, die wortwörtlich mit der Beschreibung vom Dnepr in Theatrum humanae vitae (Basileae 1604) von Theodor Zwinger, p. 4354, übereinstimmen.

42 Vetushko-Kalevich 2017, 182.

43 Eine Kopie dieses Protokolls wird jetzt in der De la Gardie-Sammlung in der Universitätsbibliothek in Tartu aufbewahrt (Almquist 1907, 238 n. 1). Widekindi 1671, 295-304 wiederholt es fast wörtlich (mit einer kurzen Einschaltung aus einer anderen Quelle), während Petrejus 1620, 463-467 eine Zusammenfassung darstellt. Man kann sich also nur wundern, dass hier Petrejus für Widekindis Quelle gehalten wird (Videkind 2000, 591 n. 305).

44 Petrejus benutzte hier die Urkunden nicht selbst, sondern verwendete ein 1619 in Hamburg ausgegebenes deutschsprachiges Itinerarium oder auszführlicher Bericht, welcher gestalt Ihre Königl. Mayest. von Schweden vnlengest Abgesandter an die... Könige, von Gross Britannien vnd Dennemarcken... verreiset... Neben drey andern newen Tractätlein: Das Eine ist ein Procesz, welcher so wol bey I. K. May. von Schweden an den Grossfürsten in Muscow gesandten Audientz am 3. Maij... am 8. Junij gehalten worden. Das Ander... 28. Junij zu Stockholm... Das Dritte ist von der Rüssen oder Moscowiter Religion, Ceremonien, Gesetzen, Policey, und Kriegeshandel, wie auch von des Landes beschaffenheit; s. Tarkiainen 1971-1972, 280. Widekindis Bericht in seinem 10. Buch ist viel ausführlicher als der von Petrejus. Er steht dem Text vom Itinerarium sehr nahe, manchmal mit auffallenden wörtlichen Übereinstimmungen; Widekindi benutzte doch nicht das Itinerarium selbst, sondern eine mit ihm gemeinsame Quelle, die er selbst als "thet widlyfftige Protocollet på Tyska", "das umfangreiche Protokoll auf Deutsch" (Widekindi 1671, 914) bezeichnet. Das Protokoll war im schwedischen Reichsarchiv aufbewahrt (Widekindi 1671, 888), aber ist verlorengegangen (Tarkiainen 1986, 193).

${ }_{45}$ Die letztere Episode ist in Petrejus 1620, 469-473 aus Schaumius 1614, E ij-E iij wörtlich abgeschrieben. 
Mit dem ziemlich knappen Material, über das wir hier verfügen - ein paar Dutzende Stellen, von denen viele nur einige Worte enthalten, und keine mehr als 250 Wörter lang ist - scheint es eine gewagte Aufgabe zu sein, diese Gleichung mit zwei Unbekannten zu lösen. Einige Beobachtungen sind uns trotzdem gelungen.

Erstens: was den Hinweis im Anhang betrifft, handelt es sich um die deutsche Ausgabe von Petrejus. Wäre das ausreichend? Vielleicht, wenn die Suche nach "inneren" Beweisen nicht zu gegensätzlichen Ergebnissen geführt hätte. Erstens gibt es Stellen in der schwedischen Ausgabe von Widekindi, wo der Text von Petrejus wortwörtlich ${ }^{46}$ wiederholt wird, nämlich der ganze Abschnitt über Marinas Flucht in III. 26 und Teile der Episoden in III. 28 und V.5. Aber auch in Abschnitten, wo Widekindis Text nur inhaltsmässig Petrejus entliehen ist, kann man Einzelheiten finden, die für die Benutzung der schwedischen Ausgabe sprechen, wie ähnlich die zwei Ausgaben von Petrejus auch sind:

\section{Petrejus 1615, II, s. 120}

Tå wille han heller lijdha then aldraslemmaste dödh... för än han på ett sådant sätt wille... förswäria sin Religion/ och föra Krijgh på sit Fädhernesland.

Petrejus 1615, II, s. 213

\section{Petrejus 1620, S. 274}

So fern er nicht köndte auff eine andere weise/ eine Fraw bekommen/ als mit verwerffung seiner Religion, vnd seines lieben Vaterlandes Vertilgung/ so wolte er... des allergrewlichsten Todes sterben.

Jstom Bascho... twingade vthi 4. Isthoma Bascho zwang binnen 4. wekors tidh öfuer 14. Slotzlän/ til Wochen 14. Häuser vnd Städte at swäria Demetrio på nytt igen.

Petrejus 1615, II, s. 255

Medhan nu thetta oenigheet således waradel drogh Her Jacob och Knäs Michael... til Knäs Wasili Zuski vthi Muskow/ och hadhe vti ett heelt åhr intaghet alla Slott och Städher som lågo emellan Finland oc Muskow/ och emellan Muskow och S. Nicolai.

Petrejus 1615, I, ss. 63-64

Petrejus 1620, S. 80-82

Skal man thet öffuerwinnal tå måste thet skee medh godha eller medh hunger.
Soll man es gewinnen/so muss es durch Hunger/ oder Kranckheit geschehen. zum eyd/ dass sie Demetrio auffs newe schweren musten.

\section{Petrejus 1620, S. 434}

Nach eroberung des hauses Mitrofwa zog der Schwedische Feldherr Graff Jacobus mit dem Reussischen Obersten... zu dem Grossfürsten in die Musscow/ vnd hetten in einem jahr alle Flecken vnd Städte/ so zwischen Finland/Musscow vnd S. Nicolai gelegen/ gezwungen.

\section{Widekindi 1672, p. 18}

Mox cum Religionem mutare et Patriae suae arma inferre nollet, omnibus illis eum privavit, et diu misera pressit servitute.

Widekindi 1672, p. 34

Hi 4. mensium spatio 14. Provinciis in obsequium redactis, urbi tandem graves incubabant.

Widekindi 1672, pp. 129-130

Hic fuit... modus solutae obsidionis Moschuae, qvae... tantum imperium ad extremum ita fatigavit, ut nulla superesset spes et redemptio ab ultima ruina, nisi per auxiliares Svecorum copias, qvae... jam omnes urbes castellaque in tractu Australi Septentrionalique inter urbem et Finlandiam, portumque S. Nicolai sita, falsae dominationis metu... liberassent.

Widekindi 1672, pp. 243-244

Castrum hoc... munitum erat... ita ut nonnisi fame vel amica transactione ad deditionem compelli posset.

46 Die Unterschiede in diesen Fällen sind meistens orthographische, aber hie und da liegen auch kleine lexikalische Veränderungen vor. Diese Stellen, sowie auch andere, wo der zwei Generationen jüngere Widekindi schwedische (ausser Petrejus urkundliche) Quellen in dem schwedischen Text etwas frei wiedergibt, wären wohl ein Schatz für einen Erforscher der schwedischen Sprachgeschichte des 17. Jahrhunderts. 
Dass Widekindi auf die deutsche Ausgabe in seiner Marginalanmerkung im Nowgorod-Anhang hinweist, bedeutet nur, dass ihm diese Ausgabe bekannt war. Es geht nicht einmal um Zitierung: der Satz "Scribunt Tyrannum de praeda ac manubiis trecentos carros preciosissimis onustos Thessauris avexisse" ist aus der ebenda erwähnten Schrift von J. C. Scaliger entlehnt, und der Hinweis auf Petrejus ist dazu berufen, diese Worte zu bekräftigen. Vielleicht fasste Widekindi Petrejus Angaben im deutschen Text ("etliche hundert Wagen", während es sich in der schwedischen Ausgabe um "öffuer 600. wagnar" handelt) als wahrscheinlichere auf. ${ }^{47}$

Die zweite Frage ist etwas komplizierter. Einerseits haben wir, wie oben erwähnt, Stellen mit wortgetreuer Wiedergabe des schwedischen Petrejus im schwedischen Widekindi. Im lateinischen Text werden sie verschieden gehandhabt: in III. 26 findet man anstatt der betreffenden Stelle nur die Marginalanmerkung: "De his clarius pluriusque Svecica aeditio"; die Stellen in III. 28 und V. 5 sind mit kleinen Verkürzungen ins Latein übersetzt.

Andererseits entspricht in den meisten Fällen der schwedische Widekindi nur inhaltsmässig dem Text von Petrejus: in fünf kompakt belegten Fällen - in II.9, III.9, III.11, III. 20 und III. 25 - kann man den entsprechenden Abschnitt ausschliesslich im lateinischen Text finden, in anderen handelt es sich um ungefähr gleichwertige Abschnitte in beiden Versionen, doch ganz ohne klare wörtliche Parallellen in Widekindis und Petrejus Schwedisch. Theoretisch gibt es hier drei Möglichkeiten: 1) der lateinische Text ist von der freien Wiedergabe von Petrejus in Widekindis schwedischem Text übersetzt; 2) die zwei Texte sind unabhängige voneinander freie Wiedergaben von Petrejus; 3 ) der schwedische Text ist vom lateinischen übersetzt.

Gegen die erste Möglichkeit sprechen folgende Beispiele (III. 28 und VI. 13):

Petrejus 1615, II, s. 255

Medhan nu thetta oenigheet således waradel drogh Her Jacob och Knäs Michael... til Knäs Wasili Zuski vthi Muskow/ och hadhe vti ett heelt åhr intaghet alla Slott och Städher som lågo emellan Finland oc Muskow/ och emellan Muskow och S. Nicolai/ och vppå alla wägar förjagat the Casacker och Poler.
Widekindi 1671, s. 159

Och war nu thenne nästan änden på then... Krijgzmachten/ som hafwer... Ryssland $i$ tu åhr så twingat/ at ingen vthwägh war at bringa thet til rätta igen/ annars/ än igenom the Swenskos vndsätning... och war nu Zahren $i$ sin frijheet åter stält/ Belägringen vphäfwin/ all then tracht på hin sijdan Muskou/ in àt Archangel/ Finland/ Lijff- och Ingermanland ifrain the Cassakers och Påhlers ströfwande Partijer frij giordt.
Widekindi 1672, pp. 129-130

Hic fuit... modus solutae obsidionis Moschuae, qvae... tantum imperium ad extremum ita fatigavit, ut nulla superesset spes et redemptio ab ultima ruina, nisi per auxiliares Svecorum copias, qvae... jam omnes urbes castellaque in tractu Australi Septentrionalique inter urbem et Finlandiam, portumque S. Nicolai sita, falsae dominationis metu ab octuaginta ferme millibus obsessorum liberassent, cunctisque itineribus ac viis Cosacos Polonosque depulissent.

"Tractu" im lateinischen Text sieht also nach einer Brücke vom schwedischen Petrejus zum schwedischen Widekindi aus, der von den 'Schlössern und Städten' schweigt, sowie unten von den 'Wegen', die dem schwedischen Petrejus und dem lateinischen Widekindi gemeinsam sind.

${ }^{47}$ Petrejus 1615, I, 57b; Petrejus 1620, 74. 
Petrejus 1615, I, s. 103

Suchana Elff hafwer sitt vrsprung Tyio mijl offuan för Wolugda aff een Springkella.
Widekindi 1671, s. 397

Mitt igenom Staden flyter denne Suchanal och kommer aldraförst 10 mijl vth om Staden vpspringandes vthur een Källa.
Widekindi 1672, p. 322

Urbs haec... lapideam arcem accepit... prope fluvium Suchana, qui 10 milliaria supra urbem ex Scaturigine oriens, per palustria loca manans, mediam praeterlabitur.

Inhaltsmässig bedeutet hier " $v$ th om" ('ausserhalb') dasselbe, denn der Fluss, der durch die Stadt fliesst, kann ja nur oberhalb der Stadt seiner Quelle entspringen, aber die formale Rückkehr zur Petrejus Formulierung bei der Annahme, Widekindis lateinischer Text sei hier von seinem schwedischen übersetzt, wäre hier etwas unerwartet.

Dazu kommen noch ein paar leichte Verschiebungen: oben haben wir "Tatariske Ödhemarcker" erwähnt, denen in Widekindis lateinischem Text "Tartarorum desertis" entspricht, im schwedischen aber das vagere "Tartariske Gebieter"; dasselbe gilt für die Beschreibung von Nöteborg, wo Petrejus von "siw starcka Rundelar" spricht, die Widekindi im lateinischen Text als "septem turritis propugnaculis" wiedergibt, auf Schwedisch aber bloss als "7 starcke Torn" ${ }^{48}$ In allen diesen Fällen ist es ganz unwahrscheinlich, dass Widekindi seinen schwedischen Text als die Vorlage für seinen nuancierten lateinischen verwenden konnte.

Theoretisch schliessen diese Beispiele die zweite Möglichkeit, d. h. dass die zwei Versionen unabhängig voneinander Petrejus folgen, nicht aus. Diese zweite Möglichkeit scheitert jedoch an mindestens drei Stellen.

Erstens II. 6:

Petrejus 1615, II, s. 225

När thetta Breffuet kom til Sandomir/ prachticerade Woiwodens Slächt och wänner myckit ther vppå/ at the måtte vpwäckia någhon ny Demetrium/ then the tå på sidstonne fingo/ och funno en vng spitzfundigh karl... Thenne instituerade the vthi alla framfarne Saker... och när han noghsampt vnderwijster war/ skickade the honom til Putimel.
Widekindi 1671, s. 47

$J$ medler tijdh draga the Sandomiriske fram then tridie Demetrium, vptänckt och anbracht til heela Rysslandz största Förderff och Vndergång; Then samma/ som han war begåfwat medh ett snält Ingenio, vnderwijsader wäl vthi gambla Historier/ och forna Tijders Kunskap.
Widekindi 1672, pp. 39-40

Interea cuditur a Sandomiriensibus fatalis ille totius Moschoviae ruinae tertius Demetrius. Ingenium docile praeteritarum rerum memoria, et artibus sinoniis instruebatur.

Das zweideutige "praeteritarum rerum", das eine wörtliche Übersetzung von "framfarne Saker" ist, soll natürlich nicht 'alte Geschichten', sondern ungefähr 'den Inhalt der vorhergehenden Episoden' bedeuten: der neue Namensbetrüger muss alles genau wissen, was er in Uglitsch 1591 und in Moskau 1606 erlitt. Die Zweideutigkeit führt zum lustigen Fehler im schwedischen Text, der folglich eine Übersetzung vom Latein darstellt.

Dann nochmals VI. 13 und der Fluss Suchona:

48 Petrejus 1615, I, 63; Widekindi 1672, 243; Widekindi 1671, 310. 
Petrejus 1615, I, s. 103

Suchana... löper mitt igenom Wolugda Stadh... och sedan åth Vstuga Stadh... Dijt kommer och en annan Elff... som heter Juga/ och falla så både tilhopa nedan för Slottet/ ther the mista sina förra nampn/ och kallas tilhopa Dwina.
Widekindi 1671, s. 397

Suchana... kommer igenom Staden/ ther hon vthwidgar sigh/ och flyter in àth Vsluga/ hwarest hon tå in om Staden blandar sigh ihoop medh floden Juga/ tå förandrandes sitt nampn och kallas Duina.

Hier fragt man sich, wie 'unter' zum "in om" = "intra" geworden ist. M. E. ist es ganz leicht zu erklären, wenn man daran denkt, wie ähnlich in der Handschrift die Buchstaben ' $t$ ' und 'f' aussehen können - man nahm einfach "infra" für "intra", sowohl bei der Übersetzung ins Schwedische als auch beim Druck des lateinischen Textes.

Noch ein, und vielleicht der interessanteste, Fall der unzweifelhaften Übersetzung vom Latein ins Schwedische findet man in V.5. Dort erzählt Widekindi die Geschichte von der Ermordung des zweiten falschen Dmitris. Laut Quellen, brachte der Sohn eines tatarischen Mursen (d. h. Edelmannes) seinen eigenen Vater in Verruf, und Dmitri liess den Vater ertränken. Der ertränkte Murse hatte jedoch Anhänger, und einer von diesen, namens Urussow (den Petrejus Roslanof nennt), versuchte den Sohn des Mursen zu töten, ermordete aber aus Versehen einen anderen Mursen. Dmitri liess ihn ins Gefängnis werfen, was, zusammen mit der Ertränkung des Mursen, Urussow veranlasste, Dmitri zu ermorden.

So, kompliziert genug, sagen es die Quellen, u. a. Petrejus, den Widekindi hier paraphrasiert und mit Kobierzycki kombiniert. ${ }^{49}$ Widekindis Darstellung sieht doch ganz verworren aus:

Widekindi 1671, ss. 262-263

Ty thet berättas at thenne Roslanof hafwer länge suttit inne i Fängelsel för ett Dråp som han oförwarandes hade begådt på en Murse/ i stället för Konungens Son $i$ Cazimou/ therföre at han hembligen hade låtit dränckia hans Fader.
Widekindi 1672, pp. 210-211

Namque referunt hunc Vrozovum diu carcere mulctatum, ob inopinam et fortuitam caedem Mursae cujusdam, quem pro Cazimoviensis Reguli filio trucidasset, quod patri proditorie necem in aqua accelerasset.

Dem lateinischen Text mangelt es, um den eigentlichen Sachverhalt auszudrücken, nur an ungefähr "quem occidere in animo habebat", welches ein Leser mit einer besonders feinen Einfühlungskraft vielleicht auch rekonstruieren kann. Der schwedische Text dagegen ist bloss eine mechanische Wiedergabe des lateinischen; das sieht man an "hans" und an "hembligen". Benutzt man hier das Pronomen "hans" anstatt "sijn", muss das Subjekt des Nebensatzes der falsche Dmitri sein, und dann wird "therföre" zu Unsinn, denn es besteht kein Ursache-Wirkungszusammenhang. Was "hembligen" ('heimlich') betrifft, so kann "proditorie" damit oft übersetzt werden, aber nur im passenden Zusammenhang: hier impliziert doch "proditorie" das Verhalten des Sohns gegenüber dem Vater, während der schwedische Übersetzer wiederum an Dmitri als Subjekt zu denken scheint und die Heimlichkeit der Ertränkung erfindet, von der die anderen Quellen schweigen.

49 Petrejus 1615, II, 262-263; Kobierzycki 1655, 319. 
Doch diese Stelle ist, sowie das oben angeführte III.28, auch dadurch interessant, dass der schwedische Text eine Übersetzung in unmittelbarer Nachbarschaft mit der Wiedergabe des originalen schwedischen Textes (in der Fortsetzung der beiden Abschnitte) darstellt; auch die Flucht von Marina befindet sich in dem Kapitel, wo schon manches aus Petrejus entlehnt ist.

Im Lichte davon, was wir über die Geschichte des Widekindi-Textes schon wissen (s. oben), ist m. E.zu vermuten, dass Widekindi Petrejus in zwei Schritten benutzte, zuerst durch Paraphrase auf Latein, dann durch den Nachtrag des schwedischen Originaltextes in die schwedische Version, in der die meisten Petrejus-Abschnitte schon als Übersetzung existierten. Dieser Nachtrag betrifft in allen drei Fällen die Kapitel, die schon einige Einzelheiten aus Petrejus enthielten.

Der Einfluss von "dem ersten schwedischen Kremlologen" auf das Werk seines Landmannes ist also ziemlich bescheiden. Von den sechs Teilen des Buches von Petrejus verwendet Widekindi nur die zwei ersten, ${ }^{50}$ und nur von Fall zu Fall, meistens wenn er die Auskünfte von Kobierzycki etwas bereichern will. Es handelt sich dabei um Einzelheiten und kurze Exzerpte, Petrejus wird von Widekindi nie, wie Kobierzycki, Seite für Seite abgeschrieben. Trotz Knappheit des Materials lässt es doch zu, festzustellen, dass Widekindi die erste, schwedische Ausgabe von Petrejus benutzte, und die Art und Weise seiner Arbeit mit diesem Text hilft uns, das Bild der Entstehungsgeschichte von Widekindis Werk zu nuancieren.

\section{Literaturhinweise}

Almquist H. Sverge och Ryssland 1595-1611: Tvisten om Estland, förbundet mot Polen, de ryska gränslandens eröfring och den stora dynastiska planen. Uppsala, Almquist \& Wiksell, 1907.

$\mathrm{AOSB}=$ Rikskansleren Axel Oxenstiernas skrifter och brefvexling. Stockholm, Kungliga Vitterhets historie och antikvitets akademien, 1888-.

Biaudet H. Gustaf Eriksson Vasa prince de Suède: Une énigme historique du 16e siècle. Helsingfors, 1913.

Bussow K. Verwirrter Zustand des Russischen Reichs, in: Bussov K. Moskovskaia khronika 1584-1613. Moskva-Leningrad, Izdatel'stvo Akademii Nauk SSSR, 1961 [Bussow K. Die Moskauer Chronik 15841613. Moskau-Leningrad, Verlag der Akademie der Wissenschaften der UdSSR, 1961], 199-327.

Geiman V. G. Sochinenie Iu. Videkinda kak istochnik po istorii Smutnogo vremeni [Geiman V. G. Das Werk von J. Widekindi als eine Quelle zur Geschichte der Zeit der Wirren], in: Videkind 2000, 509-520 (auf Russisch).

Helander H. Neo-Latin Literature in Sweden in the Period 1620-1720. Stylistics, Vocabulary and Characteristic Ideas. Uppsala, Uppsala Universitet, 2004.

Kobierzycki S. Historia Vladislai Poloniae et Sueciae Principis. Dantisci, Georgius Förster, 1655.

Kovalenko G. M., Khoroshkevich A. L., Pliguzov A. I. Apologiia Iukhana Videkinda [Kovalenko G. M., Khoroshkevich A. L., Pliguzov A. I. Eine Apologie von Johan Widekindi], in: Videkind 2000, 521-561 (auf Russisch).

Mund S. Orbis russiarum : genèse et développement de la représentation du monde "russe" en Occident à la Renaissance. Genève, Droz, 2003.

Orchard G.E. Bussow, Konrad, in: Wieczynski, J.L.(ed.) The Modern Encyclopedia of Russian and Soviet History. Vol. 6. Gulf Breeze, Academic International Press, 1978, 51-54.

Petrejus P. Regni Muschovitici Sciographia. Stockholm, Ignatius Meurer, 1615.

Petrejus P. Historien vnd Bericht von dem Grossfürstenthumb Muschkow. Lipsiae, Ex officina Bavarica, 1620.

${ }^{50}$ Eine - unsichere - Ausnahme könnte ein Detail in III. 28 sein: Jakob De la Gardie wird sein Degen nicht weggenommen, als er zur Audienz bei Wassili Schujskij eintritt, was von Petrejus im dritten Buch erwähnt wird. Widekindi könnte es jedoch De la Gardies eigenen Berichten entlehnt haben. 
Petrejus P. Een wiss och sanfärdigh berättelse, om några förandringar, som $\mathrm{j}$ thesse framledne åhr, vthi storfurstendömet Muskow skedde äre, in: Attius Sohlman, M. (red.) Stora oredans Ryssland: Petrus Petrejus ögonvittnesskildring från 1608. Stockholm, Carlssons, 1997, 83-159 (eine Faksimilausgabe).

Schaumius M. Tragoedia Demetrio-Moscovvitica. Rostock, Jochim Fuess, 1614.

Smirnov I. I. Konrad Bussov i ego khronika, in: Bussov, K. Moskovskaia khronika 1584-1613. Moskva-Leningrad, Izdatel'stvo Akademii Nauk SSSR, 1961 [Smirnov I. I., Konrad Bussow und seine Chronik, in: Bussow K. Die Moskauer Chronik 1584-1613. Moskau-Leningrad, Verlag der Akademie der Wissenschaften der UdSSR, 1961], 5-62 (auf Russisch).

Tarkiainen K. Petrus Petrejus som skildrare av Ryssland. En personhistorisk och källkritisk studie. Lychnos 1971-1972, 245-283.

Tarkiainen K. Se vanha vainooja. Helsinki, 1986.

Tarkiainen K. Petrus Petrejus. Svenskt Biografiskt Lexikon. Band 29. Stockholm, 1995-1997, 226-228.

Tarkiainen K. Petrus Petrejus - Sveriges första kremlolog, in: Attius Sohlman, M. (red.) Stora oredans Ryssland: Petrus Petrejus ögonvittnesskildring från 1608. Stockholm, Carlssons, 1997, 43-58.

Tolstikov A. V. Zachem perevodit' "Regni Muschovitici sciographia" Petra Petreia so shvedskogo iazyka [Tolstikov A. V. Warum "Regni Muschovitici sciographia" von Petrus Petrejus vom Schwedischen ins Russische übersetzen?]. Srednie veka 2011, 72, 175-186 (auf Russisch).

Tolstikov A. V. Petr Petrei v Rossii (1601-1613 gg.) [Tolstikov A.V. Petrus Petrejus in Russland (16011613)]. Studia Slavica et Balcanica Petropolitana 2012, 12, 129-142 (= Tolstikov 2012a; auf Russisch).

Tolstikov A.V. "Reliatsiia o Gustave, syne korolia Erika" i Petr Petrei, in: Grani sotrudnichestva: Rossiia $i$ Severnaia Evropa: Sbornik nauchnykh statei. Petrozavodsk, Izdatel'stvo PetrGU, 2012 [Tolstikov A.V. "Der Bericht von Gustav, dem Sohn des König Eriks" und Petrus Petrejus, in: Aspekte der Partnerschaft: Russland und Nordeuropa: eine Artikelsammlung. Petrosavodsk, Petrosavodsk Universitätsverlag, 2012], 104-121 (= Tolstikov 2012b; auf Russisch).

Vetushko-Kalevich A. Bilingual Writings on Bilingual Writings: J. Widekindi’s Letters to M. G. De la Gardie. Philologia Classica 2016, 11 (2), 289-300.

Vetushko-Kalevich A. Some Notes on the Literary Sources of "Historia Belli Sveco-Moscovitici Decennalis" by Johannes Widekindi. Philologia Classica 2017, 12 (2), 177-187.

Vetushko-Kalevich A. "Historia Vladislai" by S. Kobierzycki as a Source of "Historia Belli Sveco-Moscovitici" by J. Widekindi, in: A. Jönsson, A. Vetushko-Kalevich (eds.). Stolbovo 1617-2017: papers presented to the conference in Lund, 16th-17th February 2017 (erscheint 2018).

Videkind Iu. Istoriia desiatiletnei shvedsko-moskovitskoi voiny. Moskva, Pamiatniki istoricheskoi mysli, 2000 [Widekindi J. Die Geschichte vom zehnjärigen schwedisch-moskowitischen Krieg. Moskau, Monumente des geschichtlichen Gedankens, 2000] (auf Russisch).

Widekindi J. Thet Swenska i Ryssland Tijo åhrs Krijgz-Historie. Stockholm, Niclas Wankijff, 1671.

Widekindi J. Historia Belli Sveco-Moscovitici Decennalis. Holmiae, Nicolaus Wankijf, 1672.

Received: 11.12.2017

Final version received: 03.03.2018

\section{Уравнение с двумя неизвестными: выписки Юхана Видекинда из Петра Петрея}

\section{Арсений Анатольевич Ветушко-Калевич}

Санкт-Петербургский государственный университет,

Российская Федерация, 199034, Санкт-Петербург, Университетская наб., 7-9; st020343@student.spbu.ru

Вопрос о масштабе и характере использования шведским историографом Юханом Видекиндом в «Истории десятилетней шведско-московитской войны» хроники его соотечественника Петра Петрея до сих пор не подвергался систематическому рассмотрению; лишь несколько заимствованных пассажей были идентифицированы Хельге Альмквистом и издателями русского перевода Видекинда. Общая оценка последними значения Петрея для Видекинда несколько преувеличена. В данной статье приводится список за- 
имствований, рассматриваются проблемы различения заимствований из Петрея и заимствований из других источников - Станислава Кобержицкого, Акселя Оксеншерны, историко-географических и документальных источников. Последними Видекинд пользовался независимо от Петрея, описывающего те же события: в частности, это относится к описанию переговоров Делагарди с Бутурлиным в июне 1611 г. и к описанию приема шведских посланников в Москве в 1618 г. Уточняется, что рукописью хроники Конрада Буссова Видекинд не пользовался: в отрывках, первоисточником которых является Буссов, текст Видекинда систематически стоит ближе к тексту Петрея, который, таким образом, является промежуточным звеном традиции. В заключительной части статьи при помощи детального сопоставления всех четырех текстов (шведского и немецкого Петрея и шведского и латинского Видекинда) разрешается вопрос о механизме использования Видекиндом Петрея. Во-первых, зная о существовании немецкой версии, он тем не менее пользовался в основном шведской. Во-вторых, некоторые детали при передаче Видекиндом сведений Петрея демонстрируют, что при пересказе (а не буквальной передаче, как в трех случаях) Петрея латинский текст Видекинда первичен по отношению к его же шведскому. По-видимому, Видекинд привлекал труд Петрея в два этапа: сначала пересказывая его при составлении латинского черновика, а затем добавляя в шведский перевод этого черновика отрывки из шведского текста Петрея.

Ключевые слова: Смутное время, Ингерманландская война, шведская новолатинская литература, историография XVII в., россика, Юхан Видекинд, Пётр Петрей. 